Supporting Information for

\title{
Interchain Solvents Induced Chirality Change of 1D Helical Chains: From Achiral to Chiral Crystallization
}

\author{
Long Jiang, Xiao-Long Feng, Cheng-Yong Su, Xiao-Ming Chen, and Tong-Bu Lu*
}

The Structures of $\mathbf{4} \cdot 2 \mathrm{H}_{2} \mathrm{O}$. Four crystals of $\mathbf{4} \cdot 2 \mathrm{H}_{2} \mathrm{O}\left(\mathbf{4} \cdot 2 \mathrm{H}_{2} \mathrm{O}-1 \sim \mathbf{4} \cdot 2 \mathrm{H}_{2} \mathrm{O}-4\right)$ were selected from a single batch, the data were collected on a Bruker Smart 1000 CCD diffractometer, and the structures were solved using direct methods. The crystallographic data are summarized in Table S1. Though spontaneous resolution occurs during the crystallization of $4 \cdot 2 \mathrm{H}_{2} \mathrm{O}$, giving a chiral space group of $P 2_{1}$, the crystals are racemic twining with random values of absolute structure parameters (see Table S1).

In $4 \cdot \mathrm{H}_{2} \mathrm{O}\left(4 \cdot \mathrm{H}_{2} \mathrm{O}-1\right)$, the $\mathrm{Ni}(\mathrm{II})$ is six coordinated with four nitrogen atoms of the folded macrocyclic ligand $\mathrm{L}$, one nitrogen atom of $\left[\mathrm{Ni}(\mathrm{CN})_{4}\right]^{2-}$ and one water molecule (Figure S1a). The $\mathrm{O}(1)-\mathrm{Ni}(1)-\mathrm{N}(5)$ coordination plane and the $\left[\mathrm{Ni}(\mathrm{CN})_{4}\right]^{2-}$ plane are twisted, with dihedral angle of $55.9^{\circ}$. There are intermolecular hydrogen bonds $\left(\mathrm{O}(1) \cdots \mathrm{O}(2)=2.773(4), \quad \angle \mathrm{O}(1)-\mathrm{H}(1 \mathrm{E}) \cdots \mathrm{O}(2)=160.4 ; \mathrm{O}(1) \cdots \mathrm{N}(8)^{\mathrm{a}}=2.903(4)\right.$, $\angle \mathrm{O}(1)-\mathrm{H}(1 \mathrm{D}) \cdots \mathrm{N}(8)^{\mathrm{a}}=162.6 ; \mathrm{O}(2) \cdots \mathrm{N}(7)^{\mathrm{a}}=2.989(5), \angle \mathrm{O}(2)-\mathrm{H}(2 \mathrm{E}) \cdots \mathrm{N}(7)^{\mathrm{a}}=169.4 ;$ $\left.\mathrm{N}(2) \cdots \mathrm{N}(6)^{\mathrm{b}}=3.072(4), \angle \mathrm{N}(2)-\mathrm{H}(2 \mathrm{C}) \cdots \mathrm{N}(6)^{\mathrm{b}}=163.2 ; a=x+1, y, z ; b=x, y, z-1\right)$ between the terminal nitrogen atoms of the cyano groups with the water molecules and macrocyclic nitrogen atoms, generating hydrogen bonded 2D sheet along the ac plane 
(Figure S1b). Compound $4 \cdot \mathrm{H}_{2} \mathrm{O}-2 \sim \mathbf{4} \cdot \mathrm{H}_{2} \mathrm{O}-4$ show similar structures to that of $4 \cdot \mathrm{H}_{2} \mathrm{O}-1$. The macrocyclic ligand $\mathrm{L}$ shows $l$-configuration in the structures of $4 \cdot \mathrm{H}_{2} \mathrm{O}-1 \sim 4 \cdot \mathrm{H}_{2} \mathrm{O}-3$, while it adopts $d$-configuration in $4 \cdot \mathrm{H}_{2} \mathrm{O}-4$ (Figure $\mathrm{S} 1 \mathrm{c}$ ).

\section{The Proposed Processes for the Formation of $\Delta-3 \cdot 1.25 \mathrm{H}_{2} \mathrm{O}$ and $m e s o-2 \cdot 3 \mathrm{H}_{2} \mathrm{O}$. It's}

interesting to note that $4 \cdot 2 \mathrm{H}_{2} \mathrm{O}$ formed near the interface of the layering solution, and $\Delta-3 \cdot 1.25 \mathrm{H}_{2} \mathrm{O}$ and meso $-2 \cdot 3 \mathrm{H}_{2} \mathrm{O}$ formed above and below the interface of the layering solution, respectively. At the interface of the layering solution, neutral compound 4. $2 \mathrm{H}_{2} \mathrm{O}$ formed once $[\mathrm{Ni}(\alpha-r a c-\mathrm{L})]^{2+}$ met $\left[\mathrm{Ni}(\mathrm{CN})_{4}\right]^{2-}$. Above the interface of the layering solution, $[\mathrm{Ni}(\alpha-r a c-\mathrm{L})]\left(\mathrm{ClO}_{4}\right)_{2}$ is excessive, and $[\mathrm{Ni}(\alpha-r a c-\mathrm{L})]^{2+}$ prefers bonding to the nitrogen of $\left[\mathrm{Ni}(\mathrm{CN})_{4}\right]^{2-}$ of $\mathbf{4} \cdot 2 \mathrm{H}_{2} \mathrm{O}$ in trans position to avoid the repulsive interaction with large $[\mathrm{Ni}(f-r a c-\mathrm{L})]^{2+}$ group in $\mathbf{4} \cdot \mathrm{H}_{2} \mathrm{O}$ (Scheme $\mathrm{S} 1$ ), leading to the formation of $\left\{c i s-[\mathrm{Ni}(f-r a c-\mathrm{L})]_{2}\left[\mathrm{Ni}(\mathrm{CN})_{4}\right]\right\}^{2+}$ firstly. In the structural segment of $\left\{c i s-[\mathrm{Ni}(f-r a c-\mathrm{L})]_{2}\left[\mathrm{Ni}(\mathrm{CN})_{4}\right]\right\}^{2+}$ in $\Delta-\mathbf{3}$, the dihedral angle between the $\mathrm{N}\left(\right.$ cyano)-Ni-N(cyano) coordination plane and adjacent $\left[\mathrm{Ni}(\mathrm{CN})_{4}\right]^{2-}$ plane is $62.7^{\circ}$, which is close to corresponding dihedral angle $\left(55.9^{\circ}\right)$ in $4 \cdot 2 \mathrm{H}_{2} \mathrm{O}$, thus the $\left[\mathrm{Ni}(\mathrm{CN})_{4}\right]^{2-}$ plane of $\mathbf{4} \cdot 2 \mathrm{H}_{2} \mathrm{O}$ only needs slightly twisting during the formation from $\mathbf{4} \cdot \mathrm{H}_{2} \mathrm{O}$ to $\Delta-\mathbf{3}$. Accordingly, when $\left\{\text { cis- }[\mathrm{Ni}(f-r a c-\mathrm{L})]_{2}\left[\mathrm{Ni}(\mathrm{CN})_{4}\right]\right\}^{2+}$ reacted with $4 \cdot \mathrm{H}_{2} \mathrm{O}$ further, the $\left[\mathrm{Ni}(\mathrm{CN})_{4}\right]^{2-}$ in $\mathbf{4} \cdot \mathrm{H}_{2} \mathrm{O}$ also prefers to coordinate to $\mathrm{Ni}(\mathrm{II})$ of $\left\{\text { cis- }[\mathrm{Ni}(f-r a c-\mathrm{L})]_{2}\left[\mathrm{Ni}(\mathrm{CN})_{4}\right]\right\}^{2+}$ through its trans position to avoid the repulsive interaction between the $[\mathrm{Ni}(f-r a c-\mathrm{L})]^{2+}$ and $\left[\mathrm{Ni}(\mathrm{CN})_{4}\right]^{2-}$ (Scheme S1), generating an oligomer of $\left\{c i s-[\mathrm{Ni}(f-r a c-\mathrm{L})]_{3}\left[\mathrm{Ni}(\mathrm{CN})_{4}\right]_{2}\right\}^{2+}$. Continuous aggregation with $4 \cdot \mathrm{H}_{2} \mathrm{O}$ along the $3_{1}$ axis led to the formation of a 1D helical chain. Further connections of adjacent 
chains through the zip-like complementary interchain hydrophobic interactions gave $\Delta-3$.

Scheme S1. The proposed mechanisms for the formations of $\Delta-3$ and meso-2
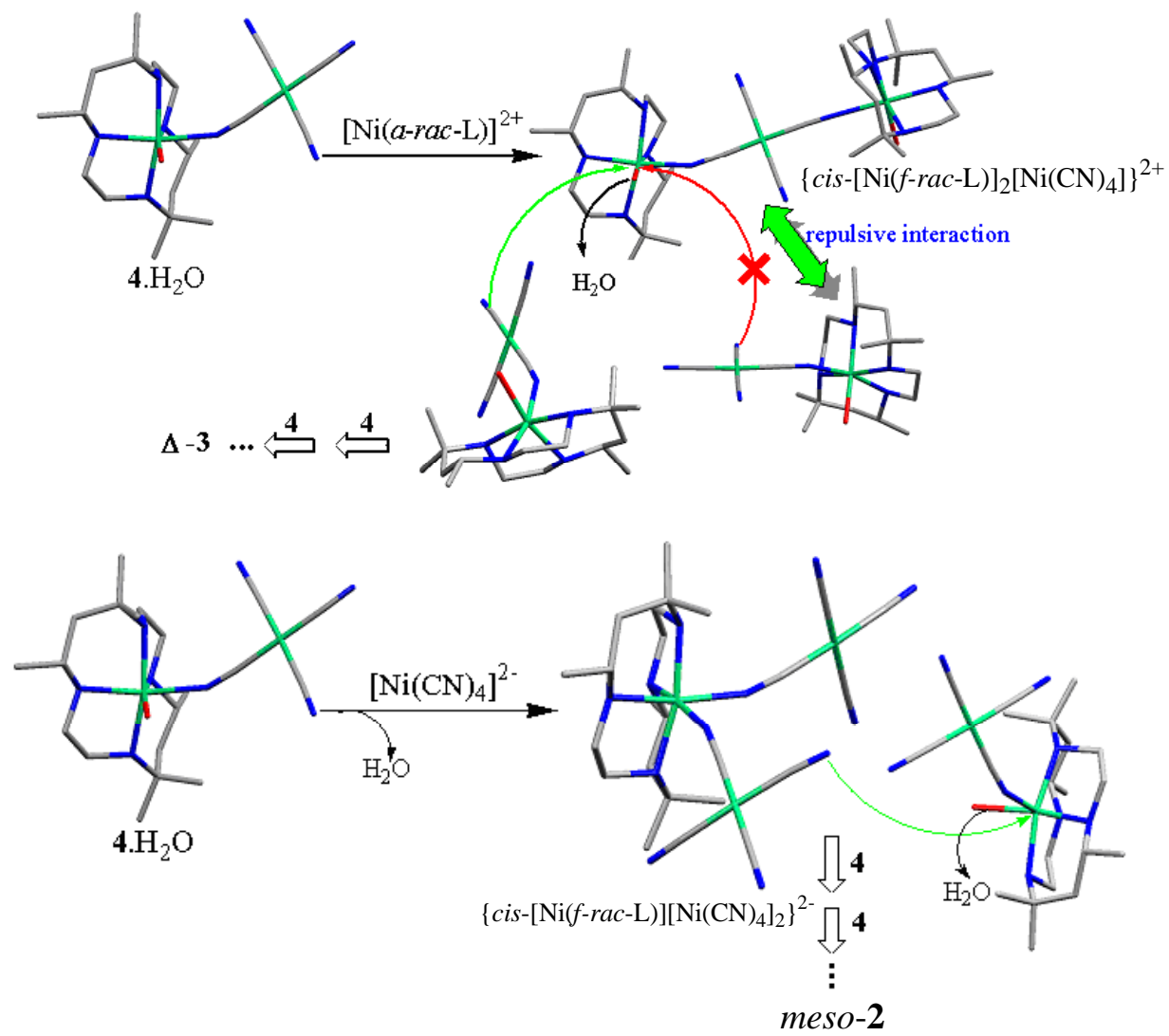

Below the interface of the layering solution, $\mathrm{K}_{2}\left[\mathrm{Ni}(\mathrm{CN})_{4}\right]$ is excessive, and $\left[\mathrm{Ni}(\mathrm{CN})_{4}\right]^{2-}$ firstly replaced the water molecule in $4 \cdot \mathrm{H}_{2} \mathrm{O}$ to generate an anion of $\left\{c i s-[\mathrm{Ni}(f-r a c-\mathrm{L})]\left[\mathrm{Ni}(\mathrm{CN})_{4}\right]_{2}\right\}^{2-} \quad($ Scheme $\quad \mathrm{S} 1)$. During the formation of $\left\{c i s-[\mathrm{Ni}(f-r a c-\mathrm{L})]\left[\mathrm{Ni}(\mathrm{CN})_{4}\right]_{2}\right\}^{2-}$, the $\left[\mathrm{Ni}(\mathrm{CN})_{4}\right]^{2-}$ plane in $4 \cdot \mathrm{H}_{2} \mathrm{O}$ was pushed to further away compared with that in $\left\{\text { cis- }[\mathrm{Ni}(f-r a c-\mathrm{L})]_{2}\left[\mathrm{Ni}(\mathrm{CN})_{4}\right]\right\}^{2+}$. Indeed, in the structural segment of $\left\{\text { cis- }[\mathrm{Ni}(f-r a c-\mathrm{L})]\left[\mathrm{Ni}(\mathrm{CN})_{4}\right]_{2}\right\}^{2-}$ in meso-2, the dihedral angle between the $\mathrm{N}\left(\right.$ cyano)-Ni-N(cyano) coordination plane and adjacent $\left[\mathrm{Ni}(\mathrm{CN})_{4}\right]^{2-}$ plane is $95.9^{\circ}$, which is larger than the corresponding dihedral angles of $62.7^{\circ}$ in $m e s o-2$ and $55.9^{\circ}$ in 
$4 \cdot \mathrm{H}_{2} \mathrm{O}$. Therefore, there is enough space to let the second $4 \cdot \mathrm{H}_{2} \mathrm{O}$ bond to $\left[\mathrm{Ni}(\mathrm{CN})_{4}\right]^{2-}$ in $\left\{c i s-[\mathrm{Ni}(f-r a c-\mathrm{L})]\left[\mathrm{Ni}(\mathrm{CN})_{4}\right]_{2}\right\}^{2-}$ at cis-position (Scheme S1), producing an oligomer of $\left\{c i s-[\mathrm{Ni}(f-r a c-\mathrm{L})]_{2}\left[\mathrm{Ni}(\mathrm{CN})_{4}\right]_{3}\right\}^{2-}$. Continuous aggregation of $\mathbf{4} \cdot \mathrm{H}_{2} \mathrm{O}$ with $\left[\mathrm{Ni}(\mathrm{CN})_{4}\right]^{2-}$ along the $2_{1}$ axis led to the formation of a $1 \mathrm{D}$ helical chain. The adjacent chains were constructed in an opposite chirality under the directions of interchain hydrogen bonds, generating an achiral isomer of meso-2.

The above proposed processes for the formations of meso-2 and $\Delta-\mathbf{3}$ are confirmed by the results of ESI-MS measurements (performed on a Thermo Finigan LCQDECA XP HPLC-MSn mass spectrometer). As shown in Figure S10a, both $\left\{[\mathrm{NiL}]_{3}\left[\mathrm{Ni}(\mathrm{CN})_{4}\right]_{2}(\mathrm{OH})(\mathrm{MeCN})\right\}^{+}$and $\left\{[\mathrm{Ni}(\mathrm{HL})]_{3}\left[\mathrm{Ni}(\mathrm{CN})_{4}\right]_{4}\left(\mathrm{H}_{2} \mathrm{O}\right)(\mathrm{MeCN})\right\}^{+}$species were formed when $4 \cdot \mathrm{H}_{2} \mathrm{O}$ was dissolved in $\mathrm{MeCN} / \mathrm{H}_{2} \mathrm{O}$ (1:1). When $[\mathrm{Ni}(\alpha-r a c-\mathrm{L})]\left(\mathrm{ClO}_{4}\right)_{2}$ was added, the excess of $[\mathrm{Ni}(\alpha-r a c-\mathrm{L})]^{2+}$ led to the formation of $\left\{[\mathrm{NiL}]_{2}\left[\mathrm{Ni}(\mathrm{CN})_{4}\right](\mathrm{OH})(\mathrm{MeCN})\right\}^{+}$species (Figure $\left.\mathrm{S} 10 b\right)$, while no $\left\{[\mathrm{NiL}]\left[\mathrm{Ni}(\mathrm{CN})_{4}\right]_{2}\right\}^{2-}$ species was found (Figure $\mathrm{S} 10 c)$. When $\mathrm{K}_{2}\left[\mathrm{Ni}(\mathrm{CN})_{4}\right]$ was added to the $\mathrm{MeCN} / \mathrm{H}_{2} \mathrm{O}(1: 1)$ solution of $4 \cdot \mathrm{H}_{2} \mathrm{O}$, the excess of $\left[\mathrm{Ni}(\mathrm{CN})_{4}\right]^{2-}$ led to the formation of $\left\{[\mathrm{NiL}]\left[\mathrm{Ni}(\mathrm{CN})_{4}\right]_{2}\right\}^{2-}$ and $\left\{\mathrm{K}[\mathrm{NiL}]\left[\mathrm{Ni}(\mathrm{CN})_{4}\right]_{2}\right\}^{-}$species (Figure $\mathrm{S} 10 d$ ), and no species related to $\left\{[\mathrm{NiL}]_{2}\left[\mathrm{Ni}(\mathrm{CN})_{4}\right](\mathrm{OH})\right\}^{+}$and $\left\{[\mathrm{NiL}]_{2}\left[\mathrm{Ni}(\mathrm{CN})_{4}\right]\right\}^{2+}$ cations were found (Figure S10e). This clear demonstrates that the excess of $[\mathrm{Ni}(\alpha-r a c-\mathrm{L})]^{2+}$ let the aggregations followed the process of formation of $\Delta-3$, while the excess of $\left[\mathrm{Ni}(\mathrm{CN})_{4}\right]^{2-}$ let the aggregations followed the process of formation of meso-2.

It's not surprise that slow conversions of the metastable compound of cis- $\left[\mathrm{Ni}(f-r a c-\mathrm{L})(\mathrm{CN})_{2}\right]$ in $\mathrm{MeCN} / \mathrm{MeOH}(1: 1)$ led to the formations of meso-2 and $\Delta-\mathbf{2}$ 
instead of $\Delta-\mathbf{3}$, since $c i s-\left[\mathrm{Ni}(f-r a c-\mathrm{L})(\mathrm{CN})_{2}\right]$ was firstly converted to a thermodynamically favorable anion of $\left[\mathrm{Ni}(\mathrm{CN})_{4}\right]^{2-}$ (Inorg. Chem. 2005, 44, 7056). Thus the existence of excessive $\left[\mathrm{Ni}(\mathrm{CN})_{4}\right]^{2-}$ let the aggregations followed the process of formation of meso-2. 
Table S1. The crystallographic data

\begin{tabular}{|c|c|c|c|c|}
\hline Compound & $4 \cdot 2 \mathrm{H}_{2} \mathrm{O}-1$ & $4 \cdot 2 \mathrm{H}_{2} \mathrm{O}-2$ & $4 \cdot 2 \mathrm{H}_{2} \mathrm{O}-3$ & $4 \cdot 2 \mathrm{H}_{2} \mathrm{O}-4$ \\
\hline formula & $\mathrm{C}_{20} \mathrm{H}_{40} \mathrm{~N}_{8} \mathrm{Ni}_{2} \mathrm{O}_{2}$ & $\mathrm{C}_{20} \mathrm{H}_{40} \mathrm{~N}_{8} \mathrm{Ni}_{2} \mathrm{O}_{2}$ & $\mathrm{C}_{20} \mathrm{H}_{40} \mathrm{~N}_{8} \mathrm{Ni}_{2} \mathrm{O}_{2}$ & $\mathrm{C}_{20} \mathrm{H}_{40} \mathrm{~N}_{8} \mathrm{Ni}_{2} \mathrm{O}_{2}$ \\
\hline fw & 542.02 & 542.02 & 542.02 & 542.02 \\
\hline temperature $(\mathrm{K})$ & $293(2)$ & $293(2)$ & $293(2)$ & 293(2) \\
\hline crystal size $(\mathrm{mm})$ & $0.49 \times 0.25 \times 0.15$ & $0.33 \times 0.28 \times 0.12$ & $0.45 \times 0.42 \times 0.16$ & $0.41 \times 0.28 \times 0.15$ \\
\hline crystal system & Monoclinic & Monoclinic & Monoclinic & Monoclinic \\
\hline space group & $P 2_{1}$ & $P 2_{1}$ & $P 2_{1}$ & $P 2_{1}$ \\
\hline$a / \AA$ & $8.8632(12)$ & $8.8452(14)$ & $8.846(2)$ & $8.8514(18)$ \\
\hline$b / \AA$ & $14.814(2)$ & $14.816(3)$ & $14.828(4)$ & $14.824(3)$ \\
\hline$c / \AA$ & $10.0634(14)$ & $10.0445(16)$ & $10.067(2)$ & $10.049(2)$ \\
\hline$\beta /^{\circ}$ & $95.152(2)$ & $95.136(3)$ & $95.138(5)$ & $95.148(4)$ \\
\hline volume $/ \AA^{3}$ & $1316.0(3)$ & 1311.1(4) & $1315.1(5)$ & $1313.2(5)$ \\
\hline$Z$ & 2 & 2 & 2 & 2 \\
\hline$D_{\mathrm{c}} / \mathrm{g} \mathrm{cm}^{-3}$ & 1.368 & 1.373 & 1.369 & 1.371 \\
\hline$\mu / \mathrm{mm}^{-1}$ & 1.462 & 1.467 & 1.462 & 1.465 \\
\hline$F(000)$ & 576 & 576 & 576 & 576 \\
\hline unique refl. $\left(R_{\text {int }}\right)$ & $5069(0.0213)$ & $5397(0.0504)$ & $5408(0.0212)$ & $5336(0.0436)$ \\
\hline parameters & 295 & 296 & 296 & 296 \\
\hline$S$ on $\mathrm{F}^{2}$ & 1.131 & 0.966 & 1.049 & 1.004 \\
\hline$R_{1},{ }^{a} w R_{2}^{b}(\mathrm{I}>2 \sigma(\mathrm{I}))$ & $0.0315,0.0643$ & $0.0563,0.1024$ & $0.0355,0.0696$ & $0.0603,0.1191$ \\
\hline$R_{1},{ }^{a} w R_{2}^{b}$ (all data) & $0.0398,0.0679$ & $0.0830,0.1287$ & $0.0475,0.0746$ & $0.1077,0.1391$ \\
\hline $\begin{array}{l}\text { Absolute structure } \\
\text { parameter }\end{array}$ & 0.00 & $0.47(2)$ & $0.482(13)$ & $0.24(3)$ \\
\hline
\end{tabular}


Table S2. Selected Bond Distances $(\AA)$ and $\operatorname{Angles}\left({ }^{\circ}\right)^{a}$

\begin{tabular}{|c|c|c|c|}
\hline \multicolumn{4}{|c|}{ meso-2.3 $\mathrm{H}_{2} \mathrm{O}$} \\
\hline $\mathrm{Ni}(1)-\mathrm{N}(1)$ & $2.155(4)$ & $\mathrm{Ni}(1)-\mathrm{N}(2)$ & $2.140(5)$ \\
\hline $\mathrm{Ni}(1)-\mathrm{N}(3)$ & $2.155(5)$ & $\mathrm{Ni}(1)-\mathrm{N}(4)$ & $2.114(5)$ \\
\hline $\mathrm{Ni}(1)-\mathrm{N}(5)$ & $2.092(5)$ & $\mathrm{Ni}(1)-\mathrm{N}(6)$ & $2.084(5)$ \\
\hline $\mathrm{Ni}(2)-\mathrm{C}(17)$ & $1.871(7)$ & $\mathrm{Ni}(2)-\mathrm{C}(18)$ & $1.858(6)$ \\
\hline $\mathrm{Ni}(2)-\mathrm{C}(19)$ & $1.865(7)$ & $\mathrm{Ni}(2)-\mathrm{C}(20)$ & $1.875(6)$ \\
\hline $\mathrm{N}(6)-\mathrm{Ni}(1)-\mathrm{N}(5)$ & $86.3(2)$ & $\mathrm{N}(6)-\mathrm{Ni}(1)-\mathrm{N}(4)$ & $88.84(18)$ \\
\hline $\mathrm{N}(5)-\mathrm{Ni}(1)-\mathrm{N}(4)$ & $168.22(19)$ & $\mathrm{N}(6)-\mathrm{Ni}(1)-\mathrm{N}(2)$ & $168.98(18)$ \\
\hline $\mathrm{N}(5)-\mathrm{Ni}(1)-\mathrm{N}(2)$ & $89.7(2)$ & $\mathrm{N}(4)-\mathrm{Ni}(1)-\mathrm{N}(2)$ & $96.97(19)$ \\
\hline $\mathrm{N}(6)-\mathrm{Ni}(1)-\mathrm{N}(1)$ & $105.63(18)$ & $\mathrm{N}(5)-\mathrm{Ni}(1)-\mathrm{N}(1)$ & $83.77(19)$ \\
\hline $\mathrm{N}(4)-\mathrm{Ni}(1)-\mathrm{N}(1)$ & $87.26(18)$ & $\mathrm{N}(2)-\mathrm{Ni}(1)-\mathrm{N}(1)$ & $84.07(17)$ \\
\hline $\mathrm{N}(6)-\mathrm{Ni}(1)-\mathrm{N}(3)$ & 84.63(19) & $\mathrm{N}(5)-\mathrm{Ni}(1)-\mathrm{N}(3)$ & $105.95(19)$ \\
\hline $\mathrm{N}(4)-\mathrm{Ni}(1)-\mathrm{N}(3)$ & $84.21(18)$ & $\mathrm{N}(2)-\mathrm{Ni}(1)-\mathrm{N}(3)$ & $86.64(18)$ \\
\hline $\mathrm{N}(1)-\mathrm{Ni}(1)-\mathrm{N}(3)$ & $166.53(19)$ & $\mathrm{C}(18)-\mathrm{Ni}(2)-\mathrm{C}(19)$ & $88.7(3)$ \\
\hline $\mathrm{C}(18)-\mathrm{Ni}(2)-\mathrm{C}(17)$ & $89.8(2)$ & $\mathrm{C}(19)-\mathrm{Ni}(2)-\mathrm{C}(17)$ & $175.1(3)$ \\
\hline $\mathrm{C}(18)-\mathrm{Ni}(2)-\mathrm{C}(20)$ & $178.8(3)$ & $\mathrm{C}(19)-\mathrm{Ni}(2)-\mathrm{C}(20)$ & $90.4(3)$ \\
\hline$C(17)-N i(2)-C(20)$ & $90.9(3)$ & & \\
\hline $\mathrm{O}(1) \cdots \mathrm{N}(7) \# 1$ & $2.914(8)$ & $\mathrm{O}(1)-\mathrm{H}(1 \mathrm{D}) \cdots \mathrm{N}(7) \# 1$ & 173.1 \\
\hline $\mathrm{O}(2) \cdots \mathrm{N}(8)$ & 2.994(9) & $\mathrm{O}(2)-\mathrm{H}(2 \mathrm{D}) \cdots \mathrm{N}(8)$ & 169.2 \\
\hline $\mathrm{O}(3) \cdots \mathrm{O}(2) \# 2$ & $2.780(7)$ & $\mathrm{O}(3)-\mathrm{H}(3 \mathrm{~A}) \cdots \mathrm{O}(2) \# 2$ & 150.9 \\
\hline $\mathrm{O}(2) \cdots \mathrm{O}(1)$ & $2.893(7)$ & $\mathrm{O}(2)-\mathrm{H}(2 \mathrm{E}) \cdots \mathrm{O}(1)$ & 165.7 \\
\hline $\mathrm{O}(3) \cdots \mathrm{O}(1)$ & $2.974(8)$ & $\mathrm{O}(3)-\mathrm{H}(3 \mathrm{~B}) \cdots \mathrm{O}(1)$ & 147.9 \\
\hline
\end{tabular}




\begin{tabular}{lclc}
\hline & \multicolumn{3}{c}{$\Delta-2 \cdot \mathrm{MeCN}$} \\
$\mathrm{Ni}(1)-\mathrm{N}(1)$ & $2.167(7)$ & $\mathrm{Ni}(1)-\mathrm{N}(2)$ & $2.141(8)$ \\
$\mathrm{Ni}(1)-\mathrm{N}(3)$ & $2.126(9)$ & $\mathrm{Ni}(1)-\mathrm{N}(4)$ & $2.112(8)$ \\
$\mathrm{Ni}(1)-\mathrm{N}(5)$ & $2.078(9)$ & $\mathrm{Ni}(1)-\mathrm{N}(6) \# 1$ & $2.108(8)$ \\
$\mathrm{Ni}(2)-\mathrm{C}(17)$ & $1.812(13)$ & $\mathrm{Ni}(2)-\mathrm{C}(18)$ & $1.854(10)$ \\
$\mathrm{Ni}(2)-\mathrm{C}(19)$ & $1.806(17)$ & $\mathrm{Ni}(2)-\mathrm{C}(20)$ & $1.849(12)$ \\
& & & \\
$\mathrm{N}(5)-\mathrm{Ni}(1)-\mathrm{N}(6) \# 1$ & $87.9(3)$ & $\mathrm{N}(5)-\mathrm{Ni}(1)-\mathrm{N}(4)$ & $169.4(3)$ \\
$\mathrm{N}(6) \# 1-\mathrm{Ni}(1)-\mathrm{N}(4)$ & $88.2(3)$ & $\mathrm{N}(4)-\mathrm{Ni}(1)-\mathrm{N}(3)$ & $84.9(3)$ \\
$\mathrm{N}(6) \# 1-\mathrm{Ni}(1)-\mathrm{N}(3)$ & $84.0(3)$ & $\mathrm{N}(6) \# 1-\mathrm{Ni}(1)-\mathrm{N}(2)$ & $169.0(3)$ \\
$\mathrm{N}(5)-\mathrm{Ni}(1)-\mathrm{N}(2)$ & $88.6(4)$ & $\mathrm{N}(3)-\mathrm{Ni}(1)-\mathrm{N}(2)$ & $86.8(3)$ \\
$\mathrm{N}(4)-\mathrm{Ni}(1)-\mathrm{N}(2)$ & $97.0(3)$ & $\mathrm{N}(6) \# 1-\mathrm{Ni}(1)-\mathrm{N}(1)$ & $106.9(3)$ \\
$\mathrm{N}(5)-\mathrm{Ni}(1)-\mathrm{N}(1)$ & $84.9(3)$ & $\mathrm{N}(3)-\mathrm{Ni}(1)-\mathrm{N}(1)$ & $166.2(3)$ \\
$\mathrm{N}(4)-\mathrm{Ni}(1)-\mathrm{N}(1)$ & $86.9(3)$ & $\mathrm{C}(19)-\mathrm{Ni}(2)-\mathrm{C}(17)$ & $176.1(7)$ \\
$\mathrm{N}(2)-\mathrm{Ni}(1)-\mathrm{N}(1)$ & $83.2(3)$ & $\mathrm{C}(17)-\mathrm{Ni}(2)-\mathrm{C}(20)$ & $90.5(5)$ \\
$\mathrm{C}(19)-\mathrm{Ni}(2)-\mathrm{C}(20)$ & $92.1(5)$ & $\mathrm{C}(17)-\mathrm{Ni}(2)-\mathrm{C}(18)$ & $89.0(4)$ \\
$\mathrm{C}(19)-\mathrm{Ni}(2)-\mathrm{C}(18)$ & $88.1(5)$ & $173.4(5)$ & \\
$\mathrm{C}(20)-\mathrm{Ni}(2)-\mathrm{C}(18)$ & & &
\end{tabular}

$\Delta-2$

$\begin{array}{ll}\mathrm{Ni}(1)-\mathrm{C}(17) & 1.842(7) \\ \mathrm{Ni}(1)-\mathrm{C}(19) & 1.872(8) \\ \mathrm{Ni}(2)-\mathrm{N}(1) & 2.155(6) \\ \mathrm{Ni}(2)-\mathrm{N}(3) & 2.144(6) \\ \mathrm{Ni}(2)-\mathrm{N}(5) & 2.068(6) \\ \mathrm{C}(17)-\mathrm{Ni}(1)-\mathrm{C}(20) & 89.3(3) \\ \mathrm{C}(20)-\mathrm{Ni}(1)-\mathrm{C}(18) & 172.8(3) \\ \mathrm{C}(20)-\mathrm{Ni}(1)-\mathrm{C}(19) & 93.2(3) \\ \mathrm{N}(5)-\mathrm{Ni}(2)-\mathrm{N}(6) & 88.0(2) \\ \mathrm{N}(6)-\mathrm{Ni}(2)-\mathrm{N}(4) & 87.2(3) \\ \mathrm{N}(6)-\mathrm{Ni}(2)-\mathrm{N}(2) & 171.2(2) \\ \mathrm{N}(5)-\mathrm{Ni}(2)-\mathrm{N}(3) & 102.7(2) \\ \mathrm{N}(4)-\mathrm{Ni}(2)-\mathrm{N}(3) & 84.4(2)\end{array}$

$\begin{array}{lc}\mathrm{Ni}(1)-\mathrm{C}(18) & 1.852(7) \\ \mathrm{Ni}(1)-\mathrm{C}(20) & 1.846(7) \\ \mathrm{Ni}(2)-\mathrm{N}(2) & 2.123(5) \\ \mathrm{Ni}(2)-\mathrm{N}(4) & 2.121(5) \\ \mathrm{Ni}(2)-\mathrm{N}(6) & 2.072(6) \\ \mathrm{C}(17)-\mathrm{Ni}(1)-\mathrm{C}(18) & 92.0(3) \\ \mathrm{C}(17)-\mathrm{Ni}(1)-\mathrm{C}(19) & 167.8(3) \\ \mathrm{C}(18)-\mathrm{Ni}(1)-\mathrm{C}(19) & 87.0(3) \\ \mathrm{N}(5)-\mathrm{Ni}(2)-\mathrm{N}(4) & 171.0(2) \\ \mathrm{N}(5)-\mathrm{Ni}(2)-\mathrm{N}(2) & 88.4(2) \\ \mathrm{N}(4)-\mathrm{Ni}(2)-\mathrm{N}(2) & 97.4(2) \\ \mathrm{N}(6)-\mathrm{Ni}(2)-\mathrm{N}(3) & 84.7(2) \\ \mathrm{N}(2)-\mathrm{Ni}(2)-\mathrm{N}(3) & 88.3(2)\end{array}$




\begin{tabular}{llll}
\hline $\mathrm{N}(5)-\mathrm{Ni}(2)-\mathrm{N}(1)$ & $85.5(2)$ & $\mathrm{N}(6)-\mathrm{Ni}(2)-\mathrm{N}(1)$ & $103.3(2)$ \\
$\mathrm{N}(4)-\mathrm{Ni}(2)-\mathrm{N}(1)$ & $88.3(2)$ & $\mathrm{N}(2)-\mathrm{Ni}(2)-\mathrm{N}(1)$ & $84.4(2)$ \\
$\mathrm{N}(3)-\mathrm{Ni}(2)-\mathrm{N}(1)$ & $168.9(2)$ & &
\end{tabular}

\section{$\Delta-3 \cdot 1.25 \mathrm{H}_{2} \mathrm{O}$}

$\begin{array}{llll}\mathrm{Ni}(1)-\mathrm{N}(1) & 2.167(3) & \mathrm{Ni}(1)-\mathrm{N}(2) & 2.115(3) \\ \mathrm{Ni}(1)-\mathrm{N}(3) & 2.197(3) & \mathrm{Ni}(1)-\mathrm{N}(4) & 2.142(3) \\ \mathrm{Ni}(1)-\mathrm{N}(5) & 2.080(3) & \mathrm{Ni}(1)-\mathrm{N}(7) \# 1 & 2.095(3) \\ \mathrm{Ni}(2)-\mathrm{C}(17) & 1.870(3) & \mathrm{Ni}(2)-\mathrm{C}(18) & 1.872(4) \\ \mathrm{Ni}(2)-\mathrm{C}(19) & 1.863(4) & \mathrm{Ni}(2)-\mathrm{C}(20) & 1.862(4) \\ \mathrm{N}(5)-\mathrm{Ni}(1)-\mathrm{N}(7) \# 2 & 86.61(12) & \mathrm{N}(5)-\mathrm{Ni}(1)-\mathrm{N}(2) & 87.86(12) \\ \mathrm{N}(7) \# 2-\mathrm{Ni}(1)-\mathrm{N}(2) & 169.90(13) & \mathrm{N}(5)-\mathrm{Ni}(1)-\mathrm{N}(4) & 172.36(13) \\ \mathrm{N}(7) \# 2-\mathrm{Ni}(1)-\mathrm{N}(4) & 88.37(12) & \mathrm{N}(2)-\mathrm{Ni}(1)-\mathrm{N}(4) & 97.92(12) \\ \mathrm{N}(5)-\mathrm{Ni}(1)-\mathrm{N}(1) & 86.23(12) & \mathrm{N}(7) \# 2-\mathrm{Ni}(1)-\mathrm{N}(1) & 104.25(13) \\ \mathrm{N}(2)-\mathrm{Ni}(1)-\mathrm{N}(1) & 83.80(13) & \mathrm{N}(4)-\mathrm{Ni}(1)-\mathrm{N}(1) & 89.43(13) \\ \mathrm{N}(5)-\mathrm{Ni}(1)-\mathrm{N}(3) & 102.72(12) & \mathrm{N}(7) \# 2-\mathrm{Ni}(1)-\mathrm{N}(3) & 84.75(13) \\ \mathrm{N}(2)-\mathrm{Ni}(1)-\mathrm{N}(3) & 88.23(13) & \mathrm{N}(4)-\mathrm{Ni}(1)-\mathrm{N}(3 & 82.53(12) \\ \mathrm{N}(1)-\mathrm{Ni}(1)-\mathrm{N}(3) & 167.80(12) & \mathrm{C}(20)-\mathrm{Ni}(2)-\mathrm{C}(19) & 90.31(16) \\ \mathrm{C}(20)-\mathrm{Ni}(2)-\mathrm{C}(17) & 88.55(15) & \mathrm{C}(19)-\mathrm{Ni}(2)-\mathrm{C}(17) & 176.76(17) \\ \mathrm{C}(20)-\mathrm{Ni}(2)-\mathrm{C}(18) & 175.9(2) & \mathrm{C}(19)-\mathrm{Ni}(2)-\mathrm{C}(18) & 89.81(17) \\ \mathrm{C}(17)-\mathrm{Ni}(2)-\mathrm{C}(18) & 91.55(17) & & \\ & & & \end{array}$

${ }^{\mathrm{a}}$ Symmetry transformations used to generate equivalent atoms: meso- $2 \cdot 3 \mathrm{H}_{2} \mathrm{O}$ : \#1 -x,-y+2,-z+1, \#2 -x,-y+1,-z+1. $\Delta-\mathbf{- 2} \cdot \mathrm{MeCN}$ : \#1 x-1/2,-y+3/2,-z. $\Delta-\mathbf{3} \cdot 1.25 \mathrm{H}_{2} \mathrm{O}: \# 1-\mathrm{x}+\mathrm{y},-\mathrm{x}+1, \mathrm{z}-1 / 3, \# 2$ $-\mathrm{y}+1, \mathrm{x}-\mathrm{y}+1, \mathrm{z}+1 / 3$. 

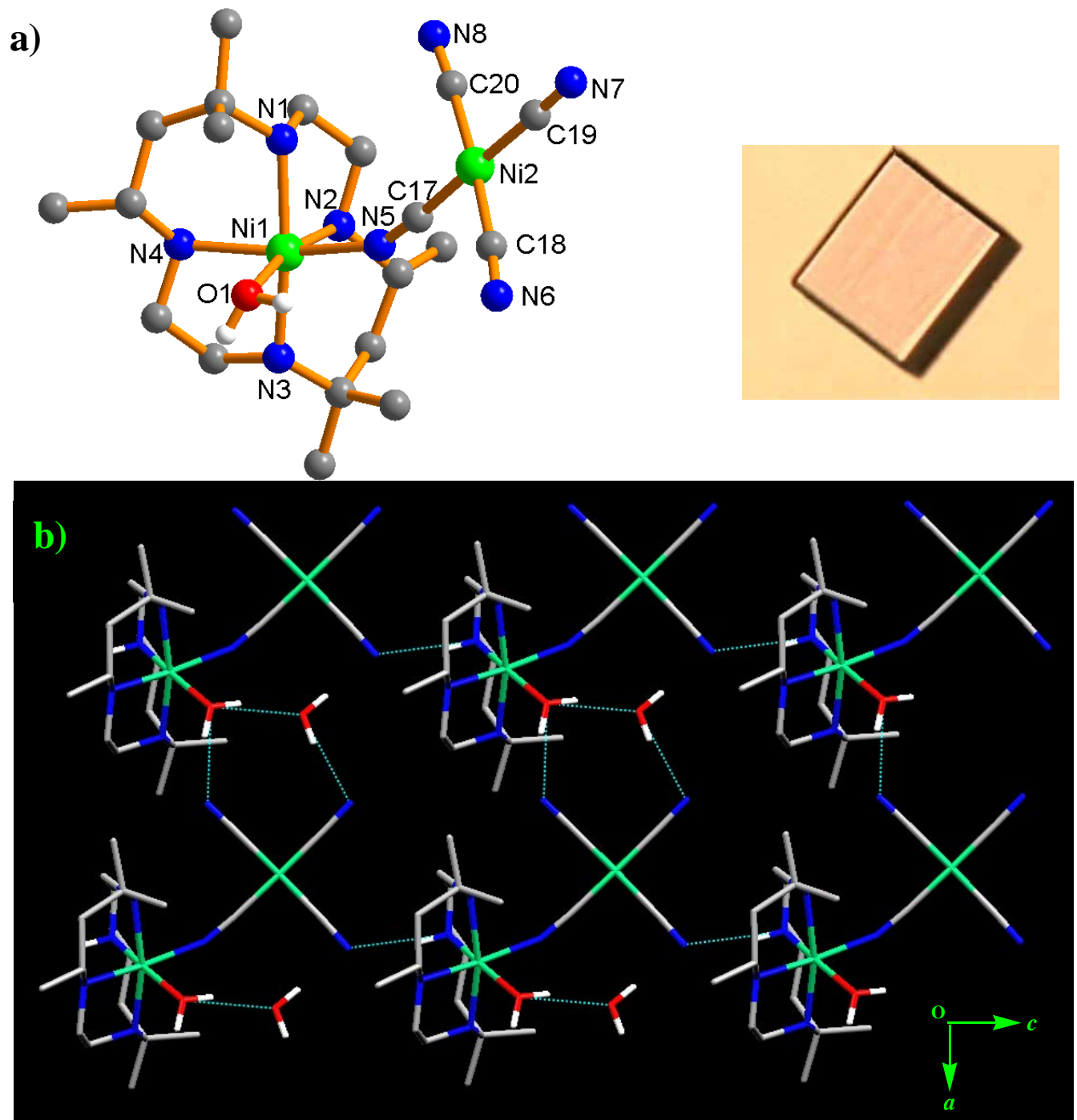

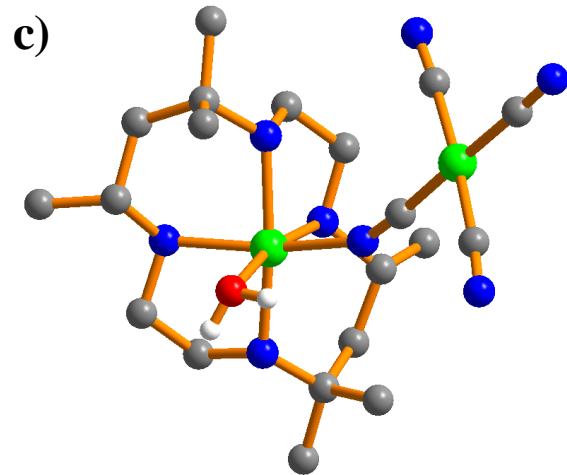

l-configuration

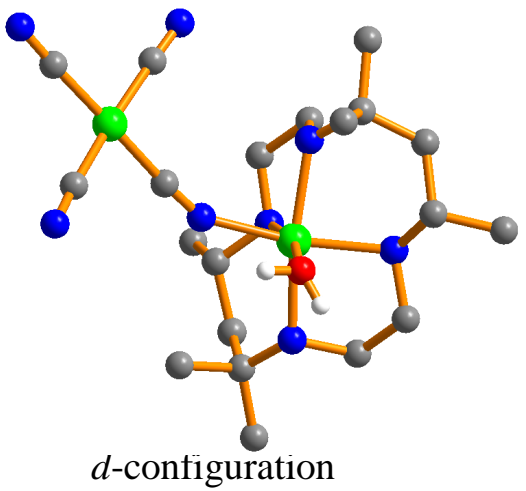

Figure S1. (a) The structure of $4 \cdot \mathrm{H}_{2} \mathrm{O}-1$ (left) and a photograph of a single crystal of $\mathbf{4} \cdot 2 \mathrm{H}_{2} \mathrm{O}$ (right).

(b) The hydrogen bonded 2D sheet along the ac plane. (c) The ligand configurations: $l$-configuration in $4 \cdot \mathrm{H}_{2} \mathrm{O}-1 \sim 4 \cdot \mathrm{H}_{2} \mathrm{O}-3$, and $d$-configuration in $4 \cdot \mathrm{H}_{2} \mathrm{O}-4$. 

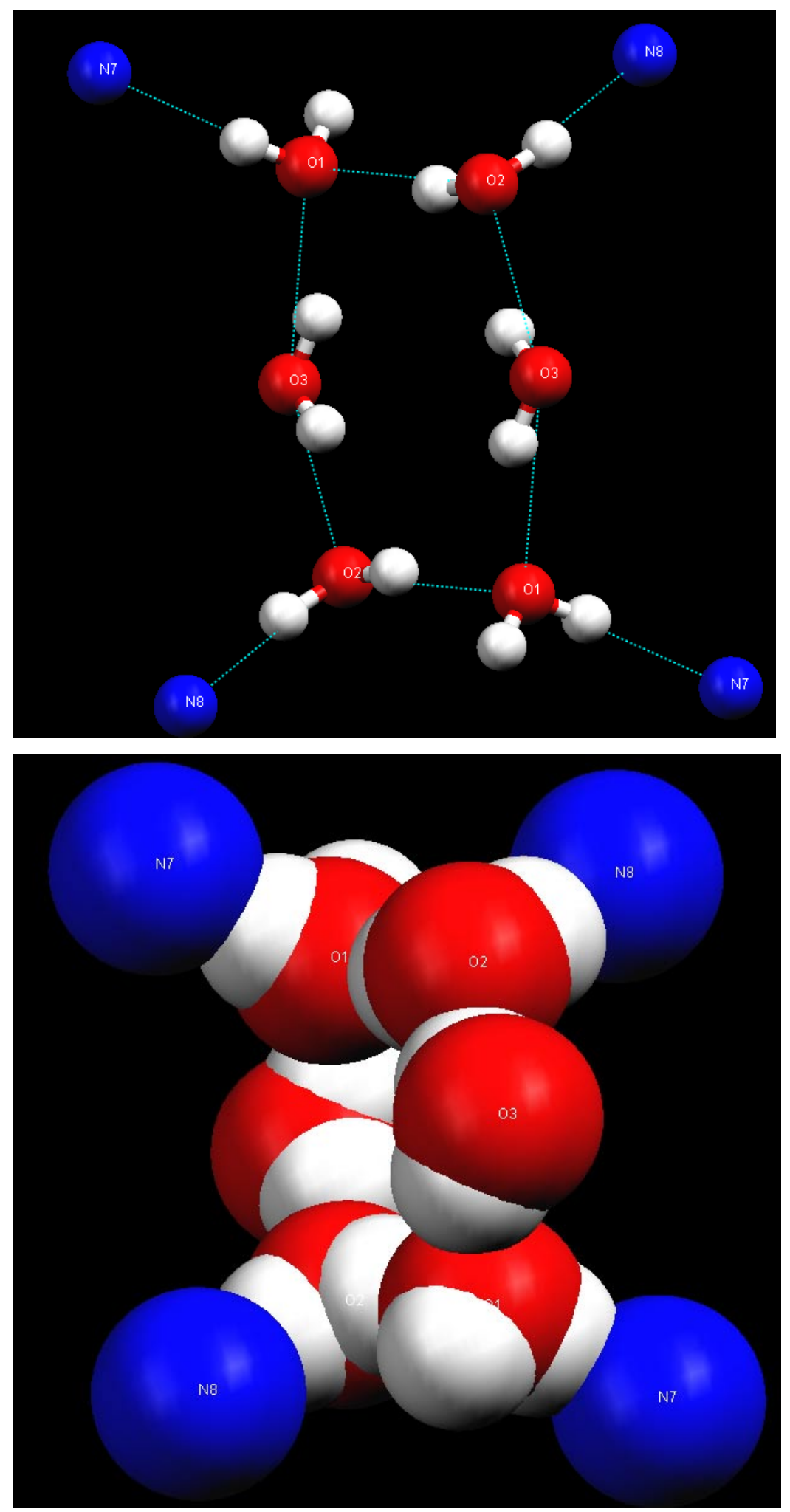

Figure S2. The hexameric water ring (top) and its space filling mode (bottom) between the $\Delta$ and $\Lambda$ helical chains in meso- $2 \cdot 3 \mathrm{H}_{2} \mathrm{O}$. 


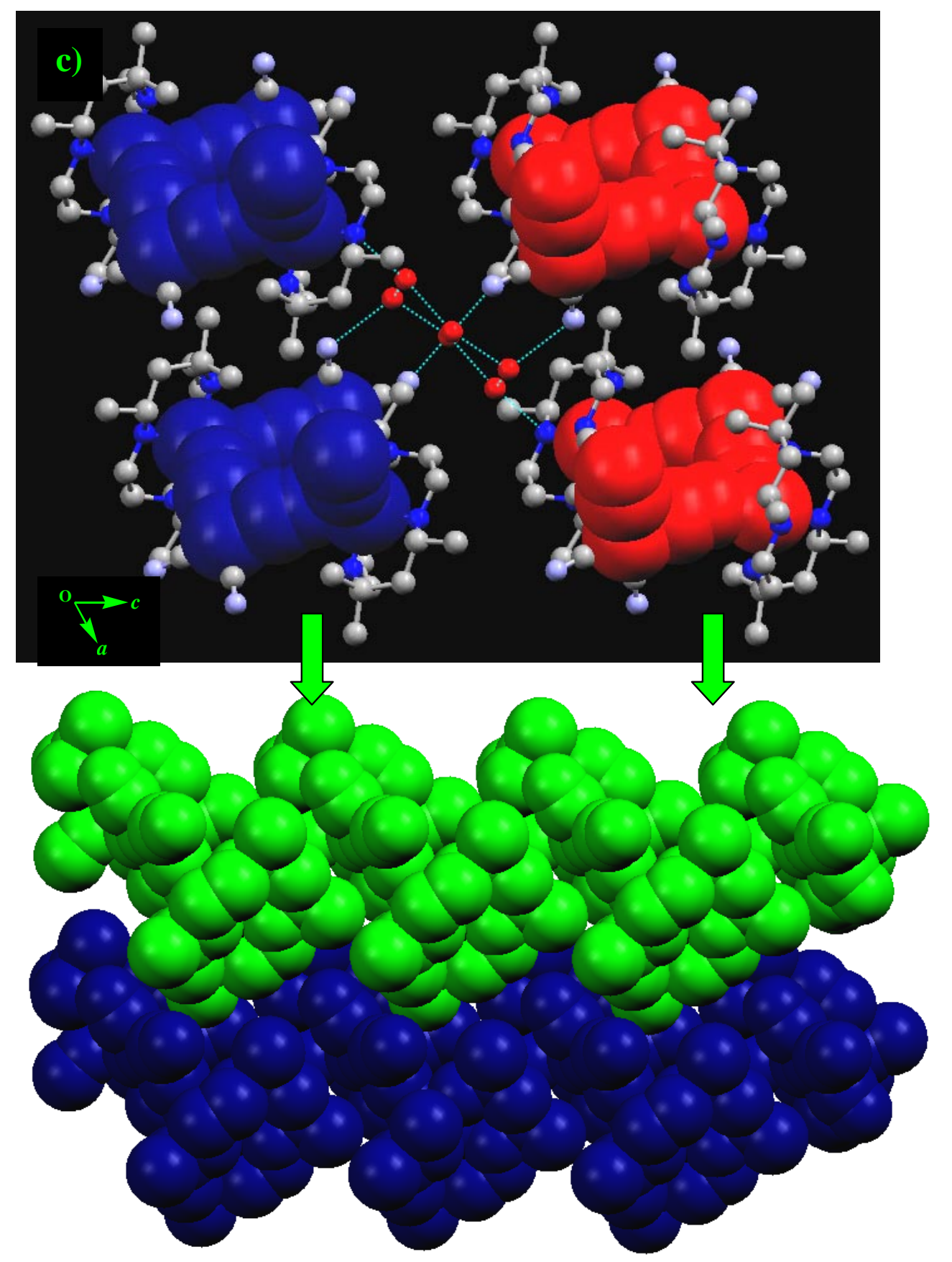

Figure S3. View of the $1 \mathrm{D}$ helical chains down the $2_{1}$ axis ( $b$ axis), showing the hexameric water clusters (red color) separated $\Delta$ (blue color) and $\Lambda$ (red color) chains (top), and the homochirality transfer of $\Delta / \Lambda$ chains along the $a$ axis through the interchain zipper-like complementary hydrophobic interactions (bottom). 
a)

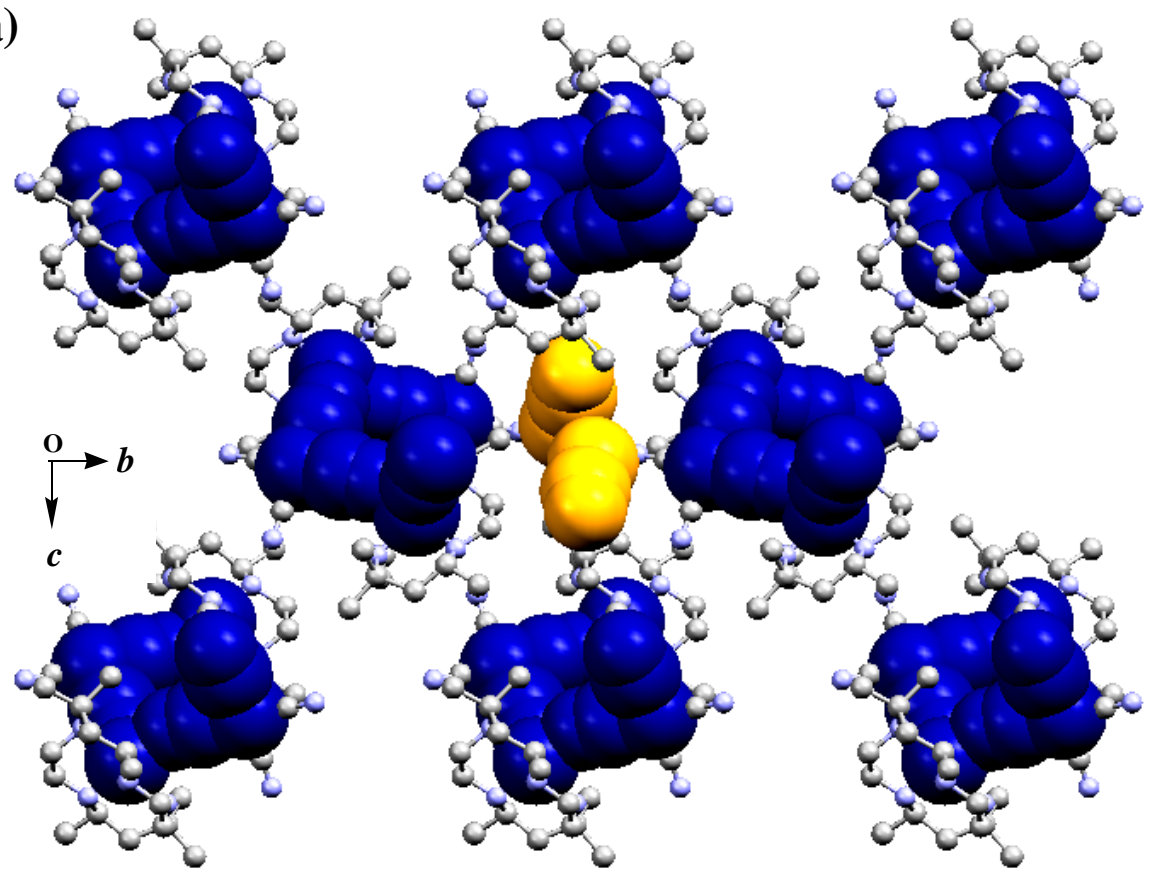

b)

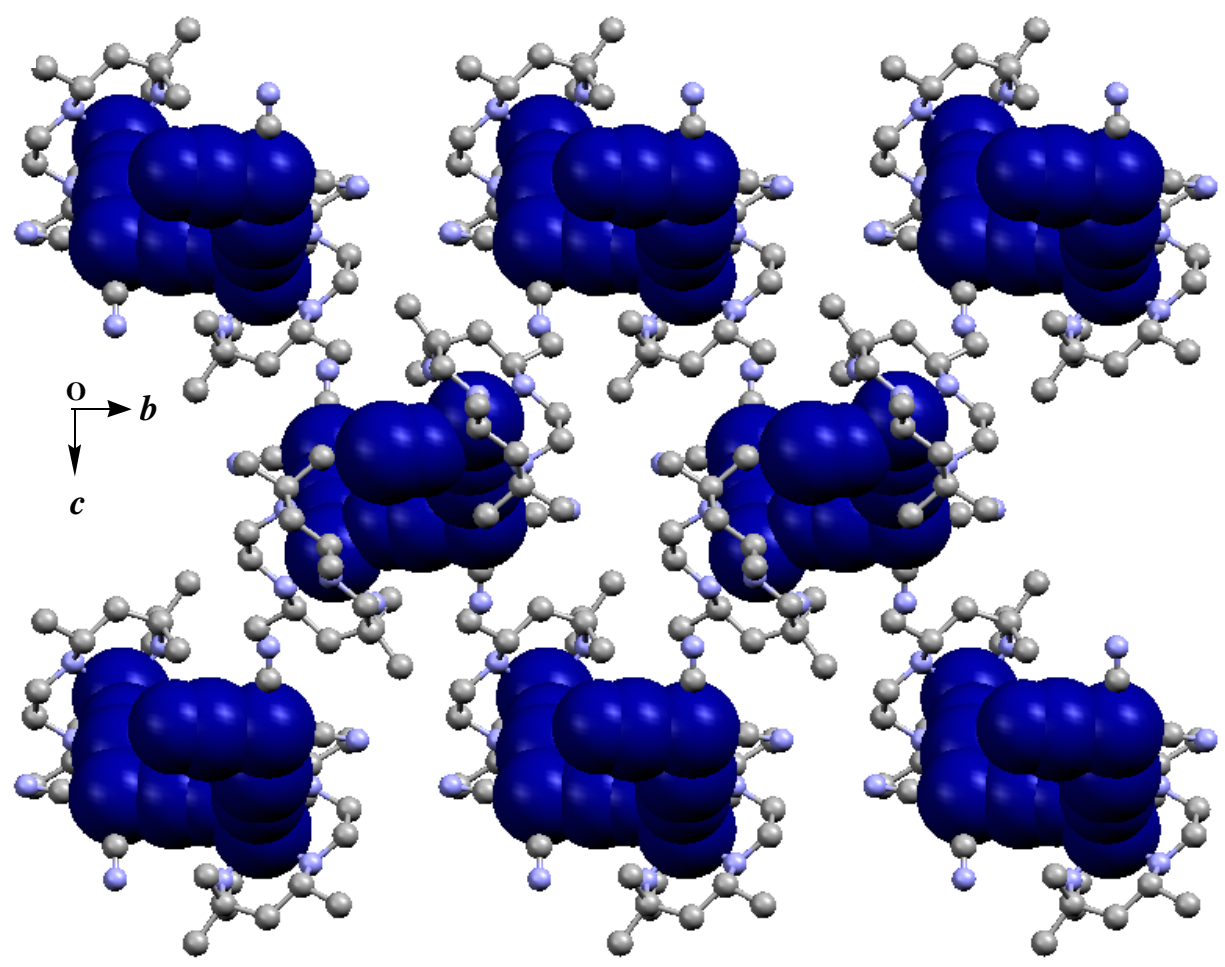

Figure S4. (a) View of the 1D right-handed helical chains along the $a$ axis, showing the homochiral structure of $\Delta-\mathbf{2} \cdot \mathrm{MeCN}$ and $\mathrm{MeCN}$ molecules (yellow color) occupied 1D narrow channels. (d) The packing arrangement of $\Delta-\mathbf{2}$ along the $a$ axis 
a)

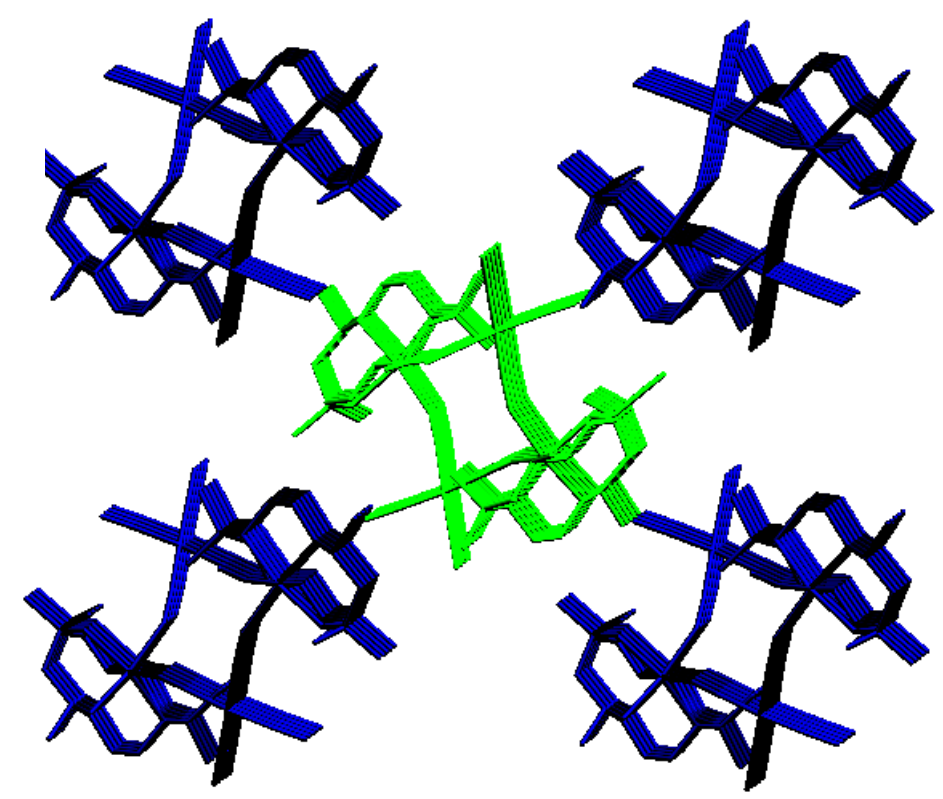

b)

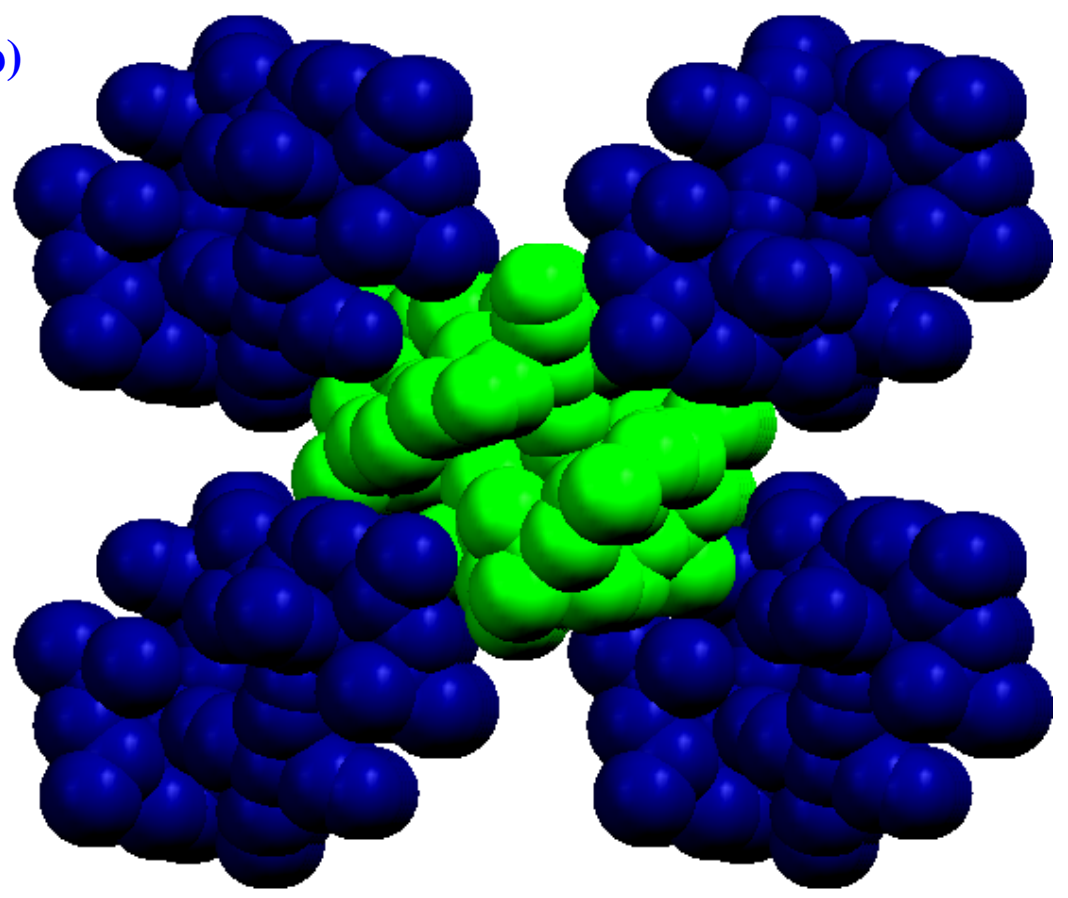

Figure S5. a) Each chain (green one) connects with four adjacent chains (blue ones) through the zip-like complementary interchain hydrophobic interactions in $\Delta-\mathbf{2} \cdot \mathrm{MeCN}$ b) The space filling mode. 

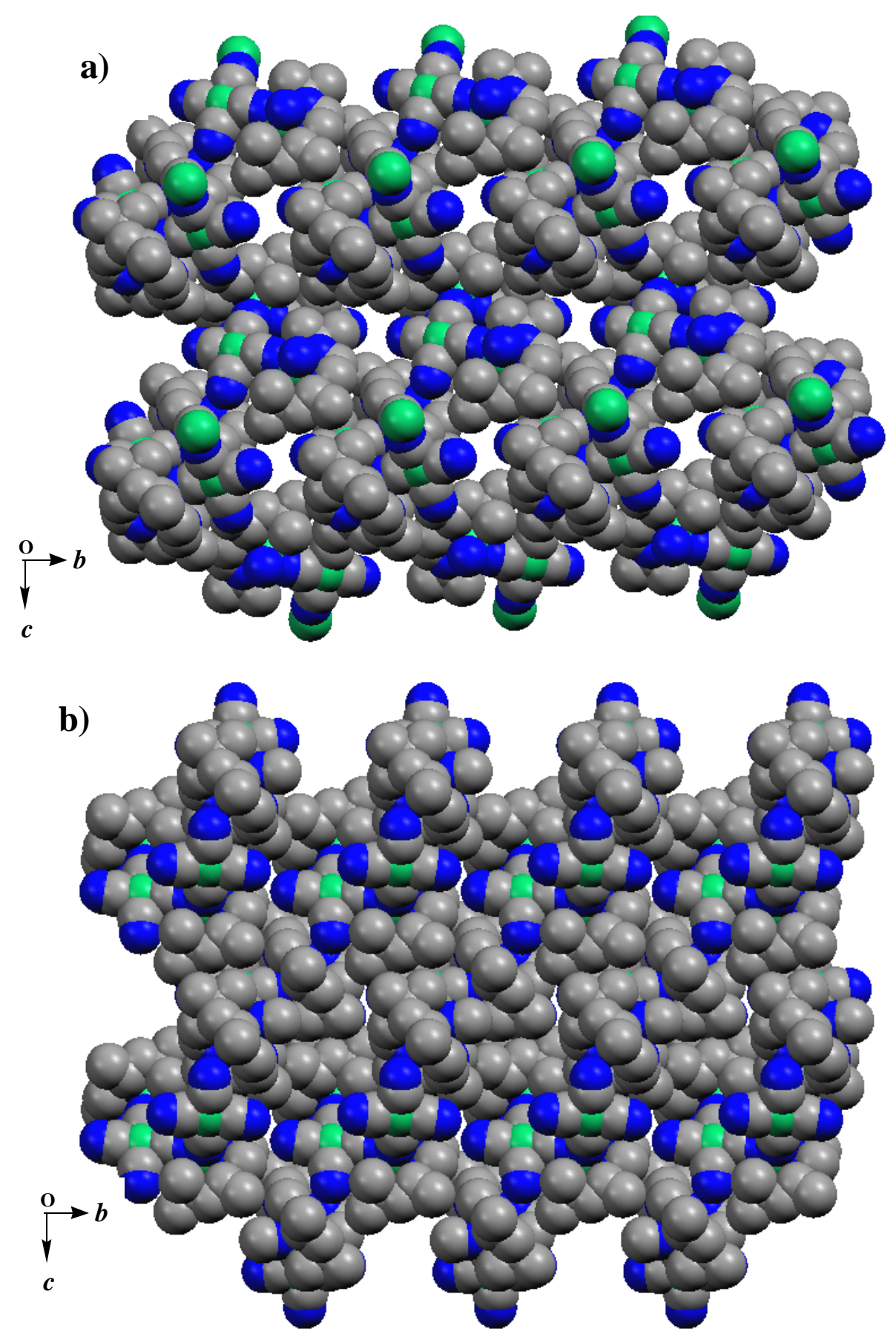

Figure S6. The space filling modes of (a) $\Delta-\mathbf{2} \cdot \mathrm{MeCN}$ and (b) $\Delta-\mathbf{2}$ along the $2_{1}$ axis, showing the narrow channels in $\Delta-\mathbf{2} \cdot \mathrm{MeCN}$ are almost disappeared after the loss of $\mathrm{MeCN}$ solvents (The $\mathrm{MeCN}$ solvents in $\Delta-\mathbf{2} \cdot \mathrm{MeCN}$ and hydrogen atoms are omitted for clarity). 
a)

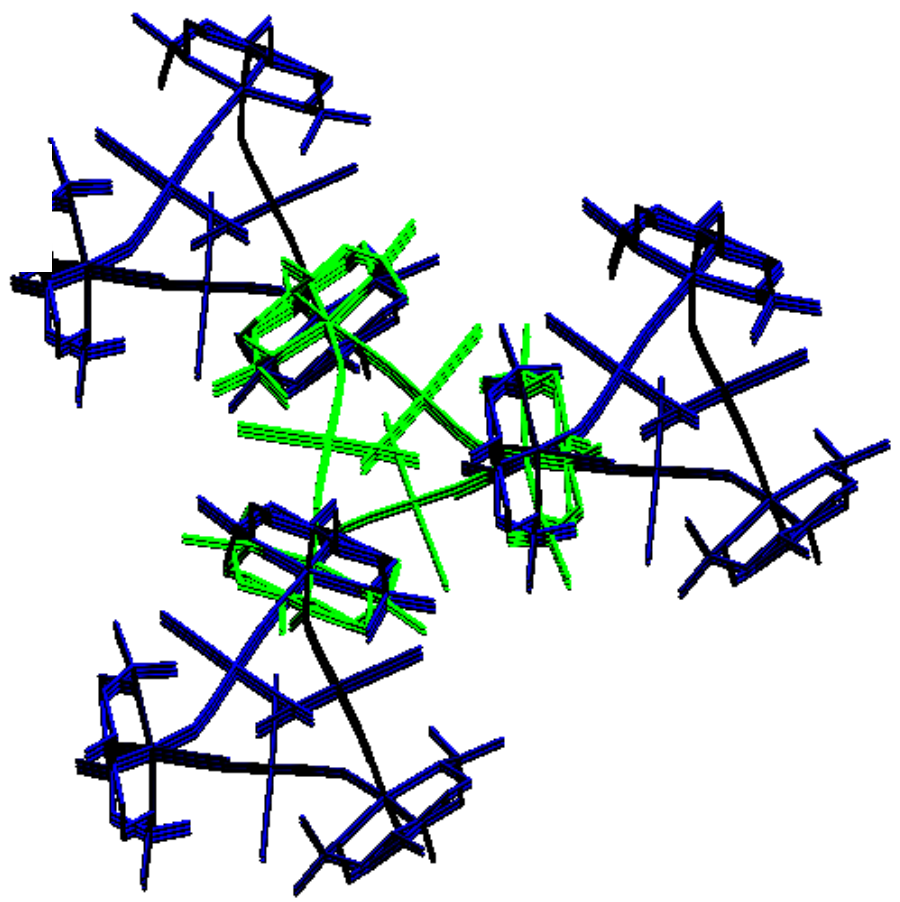

b)

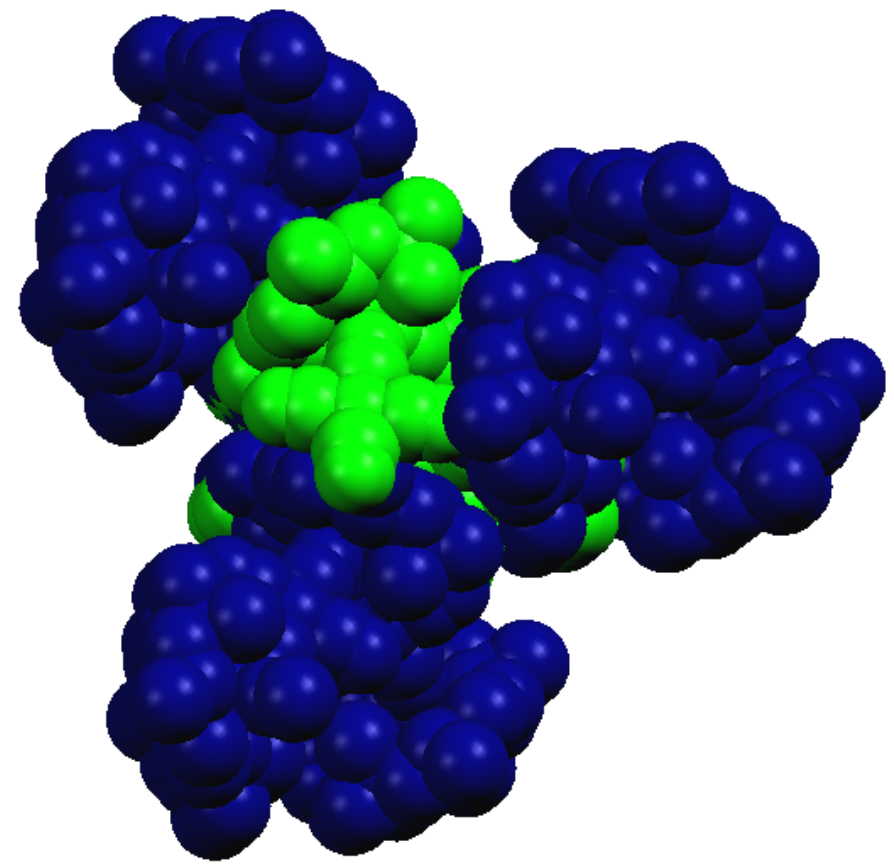

Figure S7. a) Each chain (green one) connects with three adjacent chains (blue ones) through the zip-like complementary interchain hydrophobic interactions in $\Delta-\mathbf{3} \cdot 1.25 \mathrm{H}_{2} \mathrm{O}$ b) The space filling mode. 


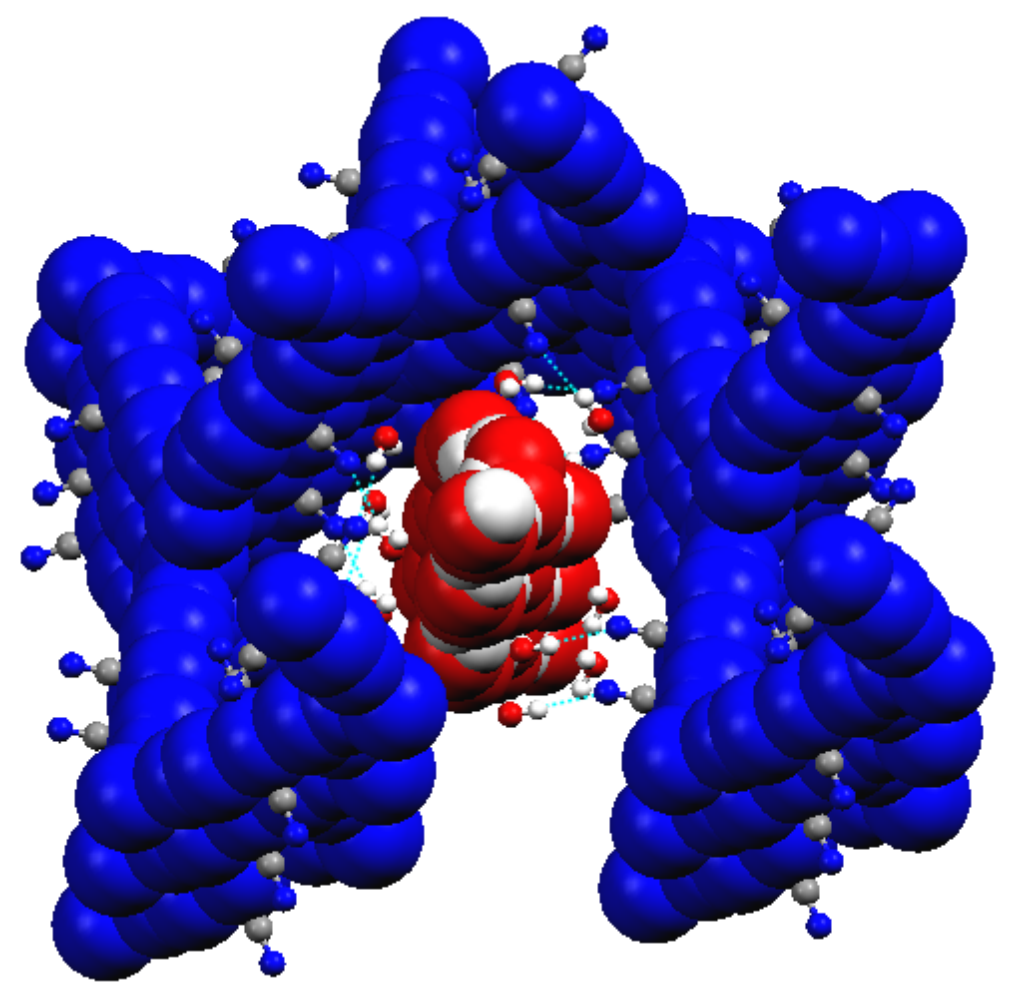

Figure S8. The 1D left-handed water chain is located in each of the 1D right-handed open channel in $\Delta \mathbf{- 3} \cdot 1.25 \mathrm{H}_{2} \mathrm{O}$ (The macrocyclic ligand was omitted for clarity).

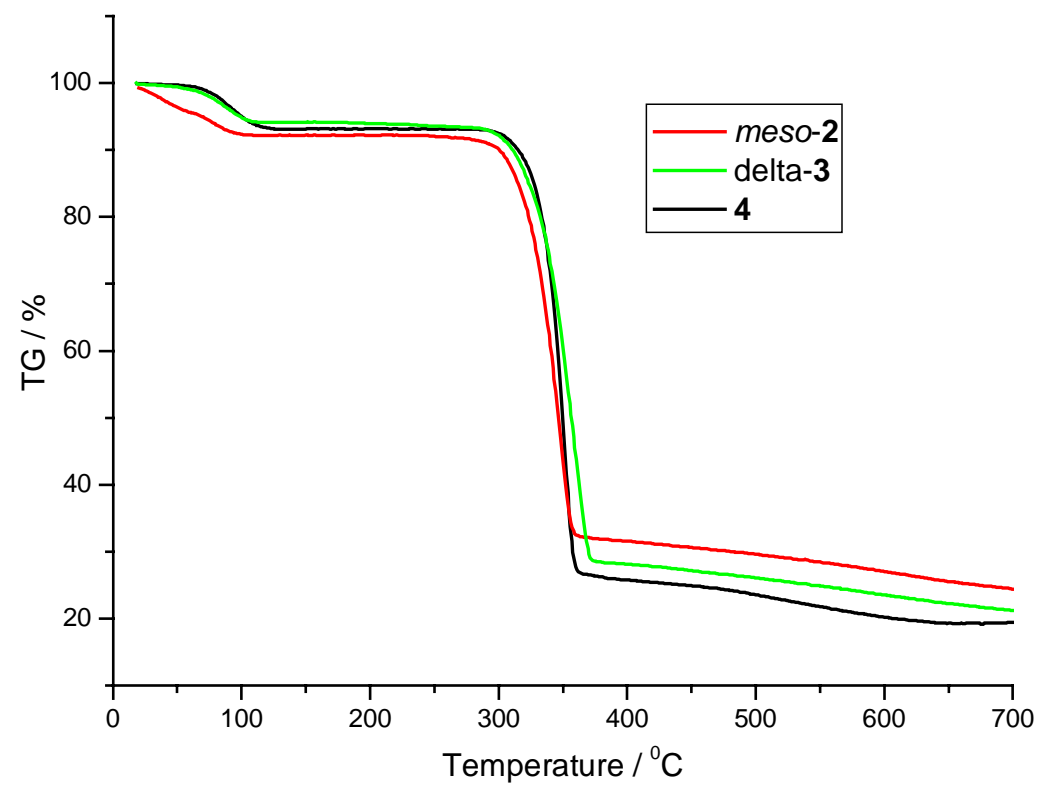

Figure S9. The TGA curves for compounds meso-2 $\cdot 3 \mathrm{H}_{2} \mathrm{O}, \mathbf{4} \cdot 2 \mathrm{H}_{2} \mathrm{O}$ and $\Delta-\mathbf{3} \cdot 1 \cdot 25 \mathrm{H}_{2} \mathrm{O}$. 

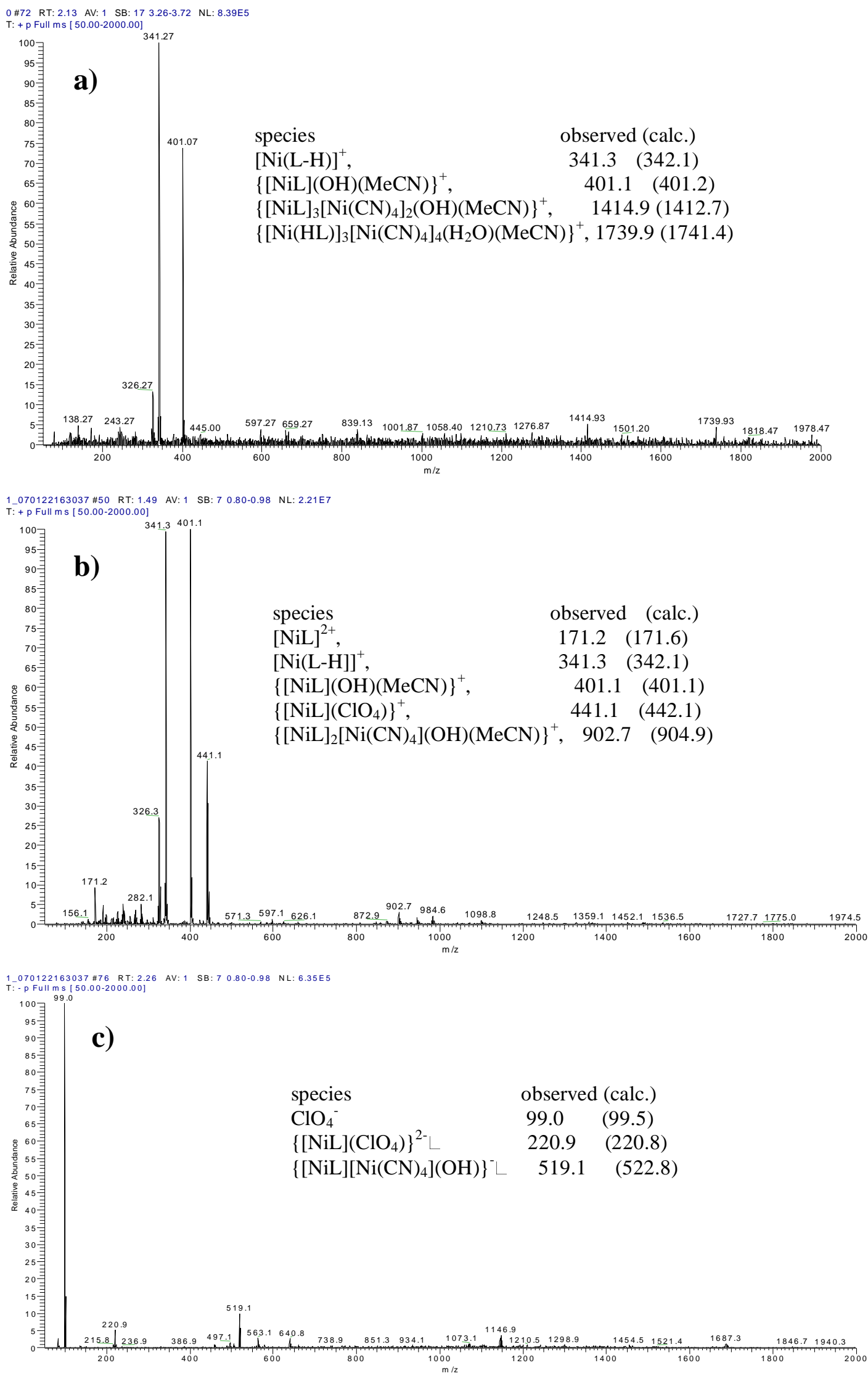

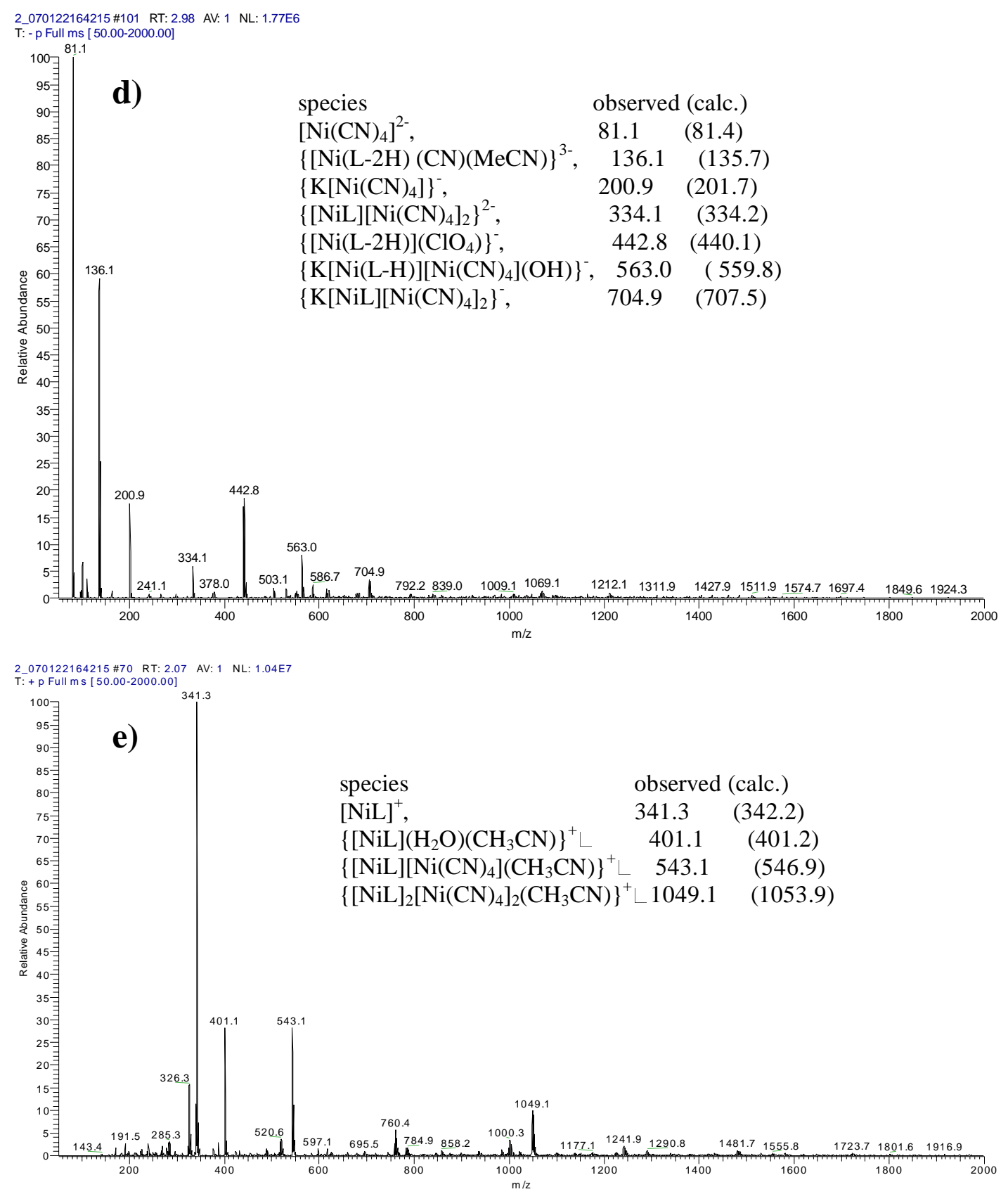

Figure S10. ESI mass spectra of $\mathbf{4} \cdot 2 \mathrm{H}_{2} \mathrm{O}$ in $\mathrm{MeCN} / \mathrm{H}_{2} \mathrm{O}(1: 1)$ measured $(a)$ after dissolving; $(b)$ and $(c)$ after adding $[\mathrm{Ni}(\alpha-r a c-\mathrm{L})]\left(\mathrm{ClO}_{4}\right)_{2} ;(d)$ and $(e)$ after adding $\mathrm{K}_{2}\left[\mathrm{Ni}(\mathrm{CN})_{4}\right]$. 